\title{
Psychological stress in Broca's versus Wernicke's aphasia
}

\author{
CLAUS HEESCHEN, JOHN RYALLS and \\ PETER HAGOORT
}

Max-Planck Institute for Psycholinguistics, Wundtiaan 1, 6525 XD Nijmegen, The Netherlands

(Received 15 June 1987; accepted 10 August 1988)

\begin{abstract}
We advance the hypothesis here that the higher-than-average vocal pitch ( $F 0)$ found for speech of Broca's aphasics in experimental settings is due, in part, to increased psychological stress. Two experiments were conducted which manipulated conversational constraints and the sentence forms to be produced by aphasic patients. Our study revealed significant differences between changes in vocal pitch of agrammatic Broca's aphasics versus those of Wernicke's aphasics and normal controls. It is suggested that the greater psychological stress experienced by the Broca's aphasics, but not by the Wernicke's aphasics, accounts for these observed differences.
\end{abstract}

\section{Introduction}

It has been found that patients with Broca's aphasia give up their typical telegraphic speech style in more formal communication -in particular, certain test situations such as reading and picture description. This change is accompanied by expressions of dissatisfaction (even curses), increased non-fluency and increased restarts and errors (Heeschen, 1985). These effects are not seen in Wernicke's aphasics. The critical factor producing this change in the Broca's aphasics seems to be the degree of freedom for expression available to the patient in the particular speaking situation. Specific tasks which impose particular sentence forms reduce the degree of freedom. Situations with more specific communication expectancies can also be anticipated to decrease the degree of freedom. Thus a continuum is formed from free conversation, which allows the patient the greatest degree of freedom, to reading, which has the least.

In three published studies in which the average vocal pitch (or fundamental frequency-F0) of read speech for Broca's aphasics has been compared to that for normal subjects, it has been found to be higher (Danly and Shapiro, 1982; Ryalls, 1984; Cooper, Soares, Nicol, Michelow and Goloskie, 1984). Table 1 shows the average fundamental frequencies derived from these studies. The overall mean difference between normal and Broca aphasic patients is $54 \mathrm{~Hz}$. Yet, in one study where the vocal frequency of Broca's aphasics and normal subjects was derived for repetition of simple three-word sentences (Ryalls, 1982) there was only a difference of $2 \mathrm{~Hz}$. Although there are now a number of additional studies which have shed some light on the suprasegmental impairment of Broca's aphasics (see Emmorey, 1987; 
Table 1. Differences in average F0 of Broca's aphasics versus normal control subjects for read speech.

\begin{tabular}{lccc}
\hline & Broca's & Normal & Difference \\
\hline Danly and Shapiro (1982) & 147 & 110 & 37 \\
Ryalls (1984) & 164 & 110 & 54 \\
Cooper et al. (1984) & 190 & 118 & 72 \\
\hline
\end{tabular}

Ryalls and Reinvang, 1986; and Gandour and Dardarananda, 1983), no research has specifically addressed the increased pitch effect of such patients.

It has been suggested that such a difference in average fundamental frequency between Broca's aphasics and normal speakers could be due to greater psychological stress on the part of the Broca's aphasics (Ryalls, 1984). If the degree of freedom in the specific conversational situation is the critical factor for inducing psychological stress, then it can be expected that the fundamental frequency (F0) of Broca's aphasics will vary accordingly. (See below for the relationship between psychological stress and fundamental frequency.) Thus it is necessary to compare the fundamental frequency of Broca's aphasics in constrained communication situations versus less constrained speech production situations.

In contrast, Wernicke's aphasics are not expected to demonstrate this effect, given their fluency and decreased concern with language difficulties (even anosognosia) (Geschwind, 1981; Lebrun, 1987). Such patients have been shown not to change their speech style in more constrained speech tasks (Heeschen, 1985; Heeschen and Kolk, 1988). Therefore they are expected to behave like normal control subjects. Thus we expect that normal control subjects and Wernicke's aphasic patients will not demonstrate higher F0 in more constrained language tasks. Although Wernicke's aphasics still monitor their speech (Schlenck, Huber and Willmes, 1987), they are unconcerned by their errors.

Starkstein and Robinson (1988) have shown that, for various reasons, Broca's aphasics show a greater tendency to depression than Wernicke's aphasics. We take this depression as a further indication of the typical Broca's aphasic's concern with his communication problem. Since the Broca patient often fails in correcting his errors, of which he is still aware, we hypothesize that he feels a situation akin to 'learned helplessness' (see Peterson and Seligman (1984) for an overview).

Conditions of increased psychological stress have been found to result in significant increases in average F0 for normal subjects (Ekman, Friesen and Scherer, 1976; Streeter, Geller and Apple, 1977). As Sapir and Aronson (1987, p. 79) have recently stated: 'the larynx is the instrument through which emotions and feelings conscious or unconscious, pleasant or unpleasant--are expressed. This fact helps to explain why the larynx is a common site for the expression of emotional conflict, stress, depression or anxiety'. From this we predict that Broca's aphasics will demonstrate a rise in $\mathrm{F} 0$ in situations where they are required to respond in some constrained manner.

In the two experiments reported here, we looked at the difference in average vocal frequency for Broca's and Wernicke's aphasics and normal control subjects across two different speech elicitation conditions-one in which the speech output is more constrained by the task, and the other which leaves the subject a greater degree of freedom. 


\section{Experiment 1}

Subjects

Eight agrammatic Broca's and eight Wernicke's aphasics were used in this experiment, as well as eight non-neurological normal controls. There were five males and three female subjects in each case. Neurological subjects were classified on the basis of their scores on the AAT (Aachener Aphasie Test-see Huber, Poek, Weniger and Willmes, 1983), which also indicates the general severity of aphasia independent of type. Subjects were also balanced for their severity of aphasia across types. Although data from both German and Dutch native speakers were used in this experiment, no language variables were manipulated according to language that could have affected the results of our experiment. The first four subjects in each aphasic group were German, while the second four in each group were Dutch. All eight normal subjects were Dutch. ${ }^{1}$

\section{Method}

Patients were familiar with the examiner and were tested in a familiar environment often their own homes. The less constrained condition consisted of subjects producing spontaneous speech in a 'coffee-time chat' conversation. In the more constrained 'experimental' condition, subjects were required to describe the actions depicted in pictures which were presented to them. They were prompted into specific sentence forms with questions such as 'What is $X$ doing?' or 'What is happening to $Y$ ?'. Thus in this condition, their speech output was more or less constrained by the topic of the actions depicted in the pictures and by the questions being asked.

Five utterances were taken from the less constrained condition and submitted to acoustic analysis. These were the first five more or less 'complete' phrases, provided they were free of environmental noise and from overlapping speech from the examiner. Single-word utterances and echolalic responses were eliminated. Although it was not required that the utterance be a complete sentence, utterances demonstrated normal sentential properties such as F0 declination (Cooper and Sorensen, 1979). It was required that utterances convey a semantically cohesive message which was not necessarily perfect syntax. Some examples are: 'Also therapy difficult' and 'The wall painted'.

These utterances were digitized at $10 \mathrm{kHz}$ and a 'peak-picking' algorithm was used to extract the fundamental frequency. A single average value was derived for each utterance by an automatic averaging procedure. Next, these five utterances were averaged to give a value for each subject, as shown in Table 2 . The ranges of the five utterances were also reported. For the 'experimental' condition, five utterances per subject were selected and measured in the same manner to arrive at an average $F 0$ for each subject in this condition.

\footnotetext{
${ }^{1}$ Dutch and German are very similar languages indeed, both phonologically and structurally, and are not expected to engage speech mechanisms such as vocal pitch in a different manner. All the properties of the experiment that were imposed on the subjects were the same in Dutch and German. The use of subjects with different linguistic backgrounds was dictated by the availability of experimental subjects.
} 
Table 2. Experiment 1 .

\begin{tabular}{|c|c|c|c|c|c|}
\hline \multirow{2}{*}{$\begin{array}{l}\text { Group } \\
(\operatorname{sex})\end{array}$} & \multicolumn{2}{|c|}{ Unconstrained } & \multicolumn{2}{|c|}{ Constrained } & \multirow[b]{2}{*}{ Difference } \\
\hline & Average & Range & Average & Range & \\
\hline \multicolumn{6}{|l|}{ Broca } \\
\hline$(\mathrm{m})$ & 116 & 7 & 104 & 38 & -12 \\
\hline (f) & 197 & 13 & 210 & 12 & +13 \\
\hline (m) & 134 & 28 & 126 & 46 & -8 \\
\hline (m) & 105 & 31 & 107 & 24 & +2 \\
\hline (f) & 176 & 18 & 194 & 25 & +18 \\
\hline (1) & 190 & 34 & 202 & 15 & +12 \\
\hline (C) & 187 & 47 & 187 & 76 & 0 \\
\hline \multirow[t]{2}{*}{ (f) } & 195 & 25 & 205 & 25 & +10 \\
\hline & & & \multicolumn{3}{|c|}{ Average Diff. Broca +4.4 (SD 10.7) } \\
\hline \multicolumn{6}{|c|}{ Wernicke } \\
\hline (f) & 164 & 26 & 159 & 19 & -5 \\
\hline (m) & 119 & 26 & 106 & 14 & -13 \\
\hline (C) & 199 & 64 & 160 & 20 & -39 \\
\hline (f) & 192 & 76 & 163 & 31 & -29 \\
\hline (f) & 209 & 62 & 216 & 50 & +7 \\
\hline (m) & 135 & 42 & 136 & 15 & +1 \\
\hline (f) & 175 & 33 & 174 & 19 & -1 \\
\hline \multirow[t]{2}{*}{ (m) } & 134 & 27 & 122 & 42 & -12 \\
\hline & & & \multicolumn{3}{|c|}{ Average Diff. Wernicke -11.4 (SD 15.7) } \\
\hline \multicolumn{6}{|l|}{ Normal } \\
\hline (f) & 166 & 108 & 139 & 42 & -28 \\
\hline (f) & 142 & 23 & 140 & 18 & -2 \\
\hline (f) & 137 & 28 & 130 & 36 & -7 \\
\hline (m) & 130 & 33 & 117 & 31 & -13 \\
\hline (m) & 138 & 52 & 123 & 4 & -15 \\
\hline (f) & 155 & 29 & 153 & 31 & -2 \\
\hline (m) & 94 & 24 & 100 & 26 & +6 \\
\hline \multirow[t]{2}{*}{ (f) } & 169 & 40 & 153 & 22 & -16 \\
\hline & & & \multicolumn{3}{|c|}{ Average Diff. Normal - 9.6 (SD 10.6) } \\
\hline
\end{tabular}

\section{Results}

The resulting values are presented in Table 2 . The difference between the averages on each of the two conditions was derived for each subject, that is the average of five tokens for the 'relaxed' condition was subtracted from the average of five tokens for the 'stressed' condition for each subject. The difference in averages between the two conditions was used because of the well-known differences in average vocal pitch between male and female speakers (symbolized by ' $m$ ' and ' $f$ ' in the tables). Differences were used, even though groups were balanced for sex. ${ }^{2}$

\footnotetext{
${ }^{2}$ The use of this difference in averages allowed us simply to consider the difference in F0 which was due to the experiment and not due to differences in male versus female fundamental frequencies (see Peterson and Barney, 1959). This means, for example, that for the purposes of our comparison a change from 90 to $100 \mathrm{~Hz}$ is treated the same as a change from $110 \mathrm{to} 120 \mathrm{~Hz}$.
} 
A one-way analysis of variance (ANOVA) with group as a factor (three levels) was conducted on the fundamental frequency difference scores. There was a significant effect for group $[F(2 \cdot 21)=3.79 ; p<0 \cdot 05]$. A Newman-Keuls post hoc test revealed a significant difference between the difference scores of the Broca patients and the Wernicke patients $(p<0.05)$. The difference scores of the Broca patients and the normal control subjects did not differ significantly from each other. Thus Broca's aphasics differ from both Wernicke patients and normal controls in that they show a higher F0 in the more constrained task, whereas Wernicke's aphasics and nonneurological controls show the opposite tendency. ${ }^{3}$

It could not be determined on the basis of these results whether the higher F0 for the Broca's aphasics in the picture-naming task was simply due to the more stressful situation of describing pictures as compared with producing spontaneous speech, or whether there was an effect of imposing sentence forms which was different in the picture description situation from what it was in spontaneous speech. To shed more light on this question, a second experiment was conducted in which the conversational demands were manipulated without changing the constraints on speech output.

\section{Experiment 2}

\section{Subjects}

Subjects were clinically evaluated using the same test as in Experiment 1 . All the subjects in this experiment were native speakers of Dutch. Four subjects who had served in Experiment 1 were included in Experiment 2. Each group (Broca's aphasics, Wernicke's aphasics and normal controls) contained eight subjects (five males and three females). Patients were again familiar with their examiner.

\section{Method}

In this experiment subjects were required to describe the various anomalies in pictures which were presented to them - for example a deer with 'horns' instead of 'antlers'. In the less constrained condition each subject was encouraged by being told it was a simple task which they would be able to perform more easily than other patients, and that it was just a practise session. For the more constrained condition, which was the same task, they were told (a) that the 'real' experiment had now begun, (b) that they were required to answer as completely as possible and (c) that their responses would now be scored. Note that the only difference between the two conditions is that the instructions set up a more constrained communication situation for the same type of stimuli. In both conditions the subjects had five pictures to

\footnotetext{
${ }^{3} \mathrm{~A}$ two-way analysis of variance with group (three levels) and condition (two levels) as factors was done on the absolute $\mathrm{F} 0$ values. This analysis revealed an overall significant effect for condition $[F(1.21)=4.69 ; p<0.05]$. However, there was also a significant group $\mathrm{x}$ condition interaction, which renders the main effect for condition uninterpretable. Naturally, the $F$ value for the group $\mathrm{x}$ condition is the same as the $F$ value for the ANOVA on the difference scores. Since this interaction was expected and has to be interpreted, we performed ANOVAs on the difference scores in both Experiments 1 and 2.
} 
describe. The pictures were counterbalanced among the subjects in each group such that all the pictures occurred both in the 'less constrained' and in the 'more constrained' conditions. This was done to eliminate potential spurious effects related to specific pictures. The less constrained condition preceded the more constrained condition. Subjects were tested in a familiar environment in a session of about 25 minutes duration.

Again the five utterances were selected under the same conditions as in Experiment 1 . The five utterances were always taken from at least two pictures, and some patients only produced one utterance per picture. These utterances were then measured to obtain an average F0 for each subject for each condition. Some examples of utterances in this experiment are: 'It's something you use at home' and 'The telephone is not'. The fundamental frequency values were obtained in the same manner as in Experiment 1.

Results

Resulting F0 averages and ranges are presented in Table 3. Just as in Experiment 1, a one-way ANOVA with group as the only factor (three levels) was effected on the F0 difference scores. There was a significant effect for group $[F(2.21=4.30 ; p<0.05]$. Post hoc Newman-Keuls tests revealed that the difference scores of the Broca's aphasics were significantly different from those of both normal controls $(p<0.05)$ and Wernicke's aphasics $(p<0.05)$. The normal controls and the Wernicke patients did not differ from each other. Thus Broca's aphasics seem to be more sensitive to the constraints of the communication situation than Wernicke's aphasics and normal control subjects.

\section{Discussion}

The results of both Experiments 1 and 2 indicate that Broca's aphasics, in contrast to Wernicke's aphasics and normal controls, react to the more formal and constrained experimental conditions with a higher F0, thereby indicating psychological stress. The constraints can be either linguistic or due to the communication situation.

Thus we have evidence that Broca's aphasics and Wernicke's aphasics react differently to the same change in experimental situation. Taking means for the differences in Experiments $I$ and 2, we find that Broca's aphasics demonstrated an overall average increase of $8.9 \mathrm{~Hz}$, while Wernicke's aphasics demonstrated an overall average decrease of $7.3 \mathrm{~Hz}$. There is, however, substantial variation from patient to patient. Such differences in reaction may reflect differences in how a patient has learned to cope with his aphasia, but these results do not allow us to address this issue. Normal control subjects reveal no overall change (i.e. $0 \mathrm{~Hz}$ ).

There are other differences in the speech of Broca's aphasics which may be correlated with their increased vocal pitch-i.e. the 'stressed' situations prevented them from using their telegraphic style. It remains to be determined how speech characteristics such as telegraphic style interact with the effect we have revealed here. Other neurological groups will have to be tested to determine the specificity of this effect to Broca's aphasia. We would also like to see more direct measures of psychological stress, such as heart rate or pupil dilation, be effected on Broca's aphasics. If our results are upheld, they may even be of potential use in speech 
Table 3, Experiment 2.

\begin{tabular}{|c|c|c|c|c|c|}
\hline \multirow{2}{*}{$\begin{array}{c}\text { Group } \\
\text { (sex) }\end{array}$} & \multicolumn{2}{|c|}{ Unconstrained } & \multicolumn{2}{|c|}{ Constrained } & \multirow[b]{2}{*}{ Difference } \\
\hline & Average & Range & Average & Range & \\
\hline \multicolumn{6}{|l|}{ Broca } \\
\hline$(\mathrm{m})$ & 105 & 19 & 138 & 21 & +33 \\
\hline (m) & 130 & 19 & 152 & 15 & +22 \\
\hline (f) & 144 & 62 & 140 & 56 & -4 \\
\hline (m) & 106 & 35 & 114 & 20 & +8 \\
\hline (m) & 125 & 20 & 138 & 40 & +13 \\
\hline (m) & 154 & 30 & 170 & 70 & +16 \\
\hline (f) & 157 & 63 & 177 & 65 & +20 \\
\hline \multirow[t]{2}{*}{ (f) } & 116 & 50 & 121 & 12 & +5 \\
\hline & & & \multicolumn{3}{|c|}{ Average Diff. Broca + 14.1 (SD 11.4) } \\
\hline \multicolumn{6}{|l|}{ Wernicke } \\
\hline$(\mathrm{m})$ & 97 & 19 & 111 & 11 & +14 \\
\hline$(\mathrm{m})$ & 112 & 19 & 104 & 34 & -8 \\
\hline (m) & 88 & 13 & 86 & 17 & -2 \\
\hline (f) & 198 & 29 & 170 & 11 & -28 \\
\hline (f) & 195 & 33 & 184 & 29 & -11 \\
\hline (f) & 173 & 10 & 184 & 64 & +11 \\
\hline (m) & 104 & 31 & 117 & 20 & +13 \\
\hline (m) & 92 & 23 & 78 & 2 & -14 \\
\hline & & & \multicolumn{3}{|c|}{ Average Diff. Wernicke -3.8 (SD 15.0) } \\
\hline \multicolumn{6}{|l|}{ Normal } \\
\hline (f) & 137 & 51 & 124 & 25 & -13 \\
\hline (f) & 143 & 27 & 140 & 44 & -3 \\
\hline (f) & 129 & 18 & 131 & 12 & +2 \\
\hline (m) & 122 & 15 & 110 & 17 & -12 \\
\hline (m) & 117 & 24 & 118 & 14 & +1 \\
\hline (f) & 154 & 40 & 165 & 38 & +11 \\
\hline (m) & 99 & 23 & 111 & 21 & +12 \\
\hline \multirow[t]{2}{*}{ (f) } & 169 & 45 & 179 & 32 & +10 \\
\hline & & & \multicolumn{3}{|c|}{ Average Diff. Normal + 2.4 (SD 19.9) } \\
\hline
\end{tabular}

therapy. These results are also of methodological relevance because they reveal that tapping into Broca's aphasics' deficit by way of highly constrained and formalized experimental situations induces psychological stress in such patients which may confound experimental results.

Finally, we are not sure that the concept of psychological stress completely accounts for the effect of higher-than-normal average vocal pitch in Broca's aphasics. Certainly the average difference of $54 \mathrm{~Hz}$ between Broca's aphasics and normal speakers in read tasks is not of the same order of magnitude as the $9 \mathrm{~Hz}$ increase we have found. Of course, a reading task is probably more stress-inducing than the simple picture description tasks used here. Also the contribution of a slight residual laryngeal spasticity to the higher average F0 on the part of the Broca's aphasics cannot be eliminated on the basis of our present findings (although this possibility has not been described, to our knowledge, in the published literature). In any case, we feel that more research should be directed to these issues in order that we may more clearly comprehend the communication deficit resulting from aphasia. 


\section{References}

Conper, W., Soares, C., Nicol, J., Michilow, D. and Goloskie, S. (1984) Clausal intonation after unilateral brain damage. Language and Speech, 27, 17-24.

COOPER, W. and Sorensen, J. (1981) Fundamental Frequency in Sentence Production (New York: Springer).

Danly, M. and Shapiro, B. (1982) Specch prosody in Broca's aphasia. Brain and Language, 16, 171-190.

Ekman, P., Friesen, W. and Scherer, K. (1976) Body movement and voice pitch in deceptive interaction. Semiotice, 16, 23-27.

Emmoriy, K. (1987) The neurological substrates for prosodic aspects of speech. Brain and Language, 30, 305-320.

Gandour, J. and Dardarananda, R. (1983) Identification of tonal contrasts in Thai aphasic patients. Brain and Language, 18, 98-114

Geschwind, N. (1981) The significance of lateralization in nonhuman species. Open peer commentary to V. H. Denneberg Hemispheric laterality in animals and the effects of carly experience. Behavioral and Brain Sciences, 4, 1-49.

HeEschen, C. (1985) Agrammatism versus paragrammatism: a fictitious opposition. In M. L. Kean (Ed), Agrammatism (New York: Academic Press).

Hefschen, C. and Kolk, H. (1988) Agrammatism and paragrammatism. Aphasiology, 2, 299-302.

Huber, W., Poeck, K., Weniger, D. and Willmes, K. (1983) Aachener Aphasie Test (Göttingen: Hogrefe).

Lebrun, Y. (1987) Anosognosia in aphasia. Cortex, 23, 251-264.

Peterson, G. and Barnit, H. L. (1959) Control methods used in a study of the vowels. Journal of the Acoustical Society of America, 24, 175-184.

Peterson, C. and Seligman, M. (1984) Causal explanation as a risk factor for depression: theory and evidence. Psychological Review, 91, 347-374.

Ryalls, J. (1982) Intonation in Broca's aphasia. Neuropsychologia, 20, $353-374$

Ryalls, J. (1984) Some acoustic aspects of CVC utterances in aphasia. Phonetica, 41, 103-111.

Ryalls, J. and Reinvang, l. (1986) Functional lateralization of linguistic tones: acoustic evidence from Norwegian. Language and Speech, 29, 389-398.

SAPIR, S. and ARONSON, S. E. (1987) Coexisting psychogenic and neurogenic dysphonia: a source of diagnostic confusion. British Journal of Communication Disorders, 22, 7380.

SChlenck, K., Huber, W. and Willmes, K. (1987) 'Prepairs' and repairs: different monitoring functions in aphasic language production. Brain and Language, 30, 226-244.

Starkstein, S. and Robinson, R. (1988) Aphasia and depression. Aphasiology, 2, 1-20.

Streeter, L., Geller, V. and Apple, W. (1977) Pitch changes during attempted deception. Journal of Personality and Social Psychology, 35, 345-350. 